\title{
Belphégor
}

Der Druck der Phantasmen: Die Illustrationen des Feuilletonromans des 19. Jahrhunderts und ihre Aneignung in Max Ernsts Collageroman Une semaine de bonté

\section{Philipp Venghaus}

\section{OpenEdition}

Journals

Édition électronique

URL : http://journals.openedition.org/belphegor/613

DOI : $10.4000 /$ belphegor. 613

ISSN : 1499-7185

Éditeur

LPCM

Référence électronique

Philipp Venghaus, « Der Druck der Phantasmen: Die Illustrationen des Feuilletonromans des 19

Jahrhunderts und ihre Aneignung in Max Ernsts Collageroman Une semaine de bonté », Belphégor [En

ligne], 13-1 | 2015, mis en ligne le 11 juillet 2015, consulté le 04 mai 2019. URL : http://

journals.openedition.org/belphegor/613; DOI : 10.4000/belphegor.613

Ce document a été généré automatiquement le 4 mai 2019.

\section{(i) $\$$}

Belphégor est mis à disposition selon les termes de la Licence Creative Commons Attribution - Pas d'Utilisation Commerciale - Pas de Modification 4.0 International. 


\title{
Der Druck der Phantasmen: Die
} Illustrationen des Feuilletonromans des 19. Jahrhunderts und ihre Aneignung in Max Ernsts Collageroman Une semaine de bonté ${ }^{1}$

\author{
Philipp Venghaus
}

As a technique, collage is a material mode of cutting and pasting distant elements - or indeed a simulation of that process. As a subversive act, it is

an instrument of détournement of pre-formed messages, "une machine à bouleverser le monde."

Louis Aragon (1921) ${ }^{2}$

1933/34 schuf Max Ernst nach La femme 100 têtes und La fille qui voulut entrer au Carmel seinen dritten Collageroman mit dem Titel Une semaine de bontés und dem Untertitel Les septs éléments capitaux. Er besteht aus 183 Collagen, eingeteilt in fünf Hefte, deren Titel, neben vorangestellten Zitaten, ${ }^{4}$ den einzigen typographischen Text der Veröffentlichung ausmachen. Nach Haupt- und Untertitel des Gesamtwerks und der Gattungsbezeichnung „Roman“ folgt auf den verschiedenfarbigen Heftumschlägen jeweils der individuelle Titel, ein dem Heft zugeordneter Tag, ein Element und ein oder mehrere Beispiele, denen eigene Kapitel gewidmet sind. Der Aufbau lässt sich am besten in Form einer Tabelle darstellen :

\begin{tabular}{|l|l|l|l|l|l|l|}
\hline Hefttitel & $\begin{array}{l}\text { Einband- } \\
\text { farbe }\end{array}$ & Tag & Element & Beispiel & $\begin{array}{l}\text { Anzahl der } \\
\text { Collagen je } \\
\text { Kapitel }\end{array}$ \\
\hline
\end{tabular}




\begin{tabular}{|c|c|c|c|c|c|c|}
\hline $\begin{array}{l}\text { Premier } \\
\text { cahier }\end{array}$ & violett & Dimanche & La boue & Le lion de Belfort & & 35 \\
\hline $\begin{array}{l}\text { Deuxième } \\
\text { cahier }\end{array}$ & grün & Lundi & L'eau & L'eau & & 27 \\
\hline $\begin{array}{l}\text { Troisième } \\
\text { cahier }\end{array}$ & rot & Mardi & Le feu & La cour du dragon & & 45 \\
\hline $\begin{array}{l}\text { Quatrième } \\
\text { cahier }\end{array}$ & blau & Mercredi & Le sang & Ædipe & & 28 \\
\hline \multirow[t]{3}{*}{$\begin{array}{l}\text { Dernier } \\
\text { cahier }\end{array}$} & gelb & Jeudi & Le noir & $\begin{array}{l}\text { Le rire du coq } \\
\text { L'île de Pâques }\end{array}$ & & $\begin{array}{l}16 \\
10\end{array}$ \\
\hline & & Vendredi & La vue & $\begin{array}{l}\text { L'intérieur de la } \\
\text { vue, Trois poèmes } \\
\text { visibles }\end{array}$ & $\begin{array}{l}\text { Premier } \\
\text { poème } \\
\text { visible } \\
\text { Deuxième } \\
\text { poème } \\
\text { visible } \\
\text { Troisième } \\
\text { poème } \\
\text { visible }\end{array}$ & $\begin{array}{l}6 \\
4 \\
2\end{array}$ \\
\hline & & Samedi & Inconnu & La clé des chants & & 10 \\
\hline
\end{tabular}

2 Wenn Werner Spies schreibt, die populären Feuilletonromane, deren Illustrationen Ernst als Grundlage seiner Collagen verwendete, seien 1933 bereits vergessen gewesen, ${ }^{5}$ so gilt dies für den einzelnen Roman. Als Genre jedoch, mit dem „die Eltern der Generation Max Ernsts Umgang hatten ", ${ }^{6}$ wie Adorno sagt, ging der roman feuilleton in die Erinnerungen der Zeitgenossen Max Ernsts ein. Die narrativen Muster und Bilder des Feuilletonromans, die nach Breton „wohl mehr als alles andere das besondere wesen unserer träume [...] bestimmen ", prägten noch das kollektive Unbewusste der jungen Menschen, die in den Ersten Weltkrieg zogen.

3 Form und Inhalt des Feuilletonromans sind eng mit den technischen Mitteln und wirtschaftlichen Bedingungen seiner Drucklegung verwoben. Im 19. Jahrhundert wird die Zeitung zum Hauptmedium für die massenhafte Verbreitung populärer Literatur. Der Serienroman steht am Beginn moderner Presse und Kultur. ${ }^{8}$ Die Produktionsbedingungen des Feuilletonromans, auf die uns der Collageroman verweist, sind nicht zu trennen von den Produktionsbedingungen der Mythen und Phantasmen, von denen die Illustrationen zeugen: Eine Welt, die sich einerseits von mythischen Mächten bedroht sieht, in der andererseits Menschen und Dinge industriell rationalisierter Verwertung unterworfen sind; eine Unterwerfung, die ihren Exzess im industrialisierten Weltkrieg erfährt.

4 In Ernsts ungewöhnlicher künstlerischer Aneignung der Feuilletonromane stehen Bilder von Verbrechen und Gewalt im Vordergrund. Es liegt nahe, darin weniger eine Verherrlichung von Gewalt, Trieb und Unbewusstem, als eine Reflexion auf die Vorgeschichte des Faschismus zu sehen: eine Kritik des in der Zwischenkriegszeit starken Bedürfnis es die eigene Distinguiertheit zur Schau $\mathrm{zu}$ tragen, diesem „Unterscheidungswillen“ wie es Lethen nennt;" ein Angriff auf die faschistischen FreundFeind-Logik; ein Versuch, die Absurdität der damit verbundenen Einteilung in gerechte 
und böse Gewalt vorzuführen. Die Aneignung der Feuilletonromanillustration im Collageroman und damit eines der bedeutendsten „populären“ Genres des 19. Jahrhunderts für das Publikationssystem Kunst geht viel weiter als ein Sich-bedienen in einem Fundus spektakulären Bildmaterials.

Der Collageroman präsentiert den Feuilletonroman als wichtigen Spiegel seiner Zeit, doch macht er ihn - das wird durch die Verfremdung klar - nicht als realistisches, sondern als phantasmatisches Genre lesbar. Die technisch aufwendige Verarbeitung, bei der sich der Collageroman über die Illustrationen hinaus auch in seinem Erscheinungsbild und in seiner Publikationsweise charakteristische Merkmale des Feuilletonromans aneignet, würdigt diesen als Meilenstein in der Entwicklung künstlerischer Techniken. „Populäre“ und „hohe“ Kunst begegnen sich. Dass dieses Aufeinandertreffen einer Kollision gleicht, die keine der beiden unverletzt lässt, legt das bestimmende Prinzip der Collage nahe.

\section{Produktionsbedingungen des Feuilletonromans und seiner Illustrationen}

6 In der äußeren Gestaltung und der Publikationsweise des Collageromans bedient sich Ernst der Tradition des Feuilletonromans. Ernst nannte sein Collagenensemble „Roman“. Der Untertitel Les sept éléments capitaux spielt auf die sieben Todsünden an $;^{10}$ eine ältere Bedeutung von capital, „qui coûte la tête“ ", ${ }^{11}$ bestärkt dies. Selbst wenn Ernst Les sept péchés capitaux von Eugène Sue nicht kannte, besteht ein klarer Bezug zu populären Titeln, in denen mit „la faute, le péché, ou le crime“ geworben wurde. ${ }^{12}$ Auch die Schrift der Hefttitel erregt Aufmerksamkeit, da sie einer verbreiteten "Typographie des Horrors“ folgt.

7 In der Zuordnung eines Wochentags zu jedem Heft kann eine Anspielung auf das wöchentliche Erscheinen vieler Feuilletons gesehen werden. ${ }^{13}$ Wie ein Serienroman sollten Max Ernsts Collagen ursprünglich in sieben nacheinander gelieferten Heften erscheinen. Mit ihrer festen Bindung und ihrem Format, 28,58 x 23,18 cm, erinnern die Hefte an die gebundenen Romansammlungen, die in der zweiten Hälfte des 19. Jahrhunderts oft herausgegeben wurden, nachdem ein Roman erfolgreich in einer Tagesoder Wochenzeitung veröffentlicht worden war.

Offensichtlich fanden sich jedoch, trotz verschiedener Anzeigen, unter anderem in der Zeitschrift Minotaure, und selbst nachdem bereits die ersten zwei Hefte im April 1934 im Verlag der Galeristin Jeanne Bucher erschienen waren, nicht die erhofften Subskribenten, sodass bereits in einer Anzeige im Mai von nur noch sechs Heften die Rede ist. ${ }^{14}$ Schließlich wurden sogar die letzten drei Tage in einem fünften und letzten Heft zusammengefasst, das erst im Dezember erschien. ${ }^{15}$

Max Ernsts Collagen dienten als Vorlagen für Strichätzungen, die bei Georges Duval in Paris in 800 Exemplaren auf „Papier Navarre“ gedruckt wurden. Dazu sollten laut der Anzeige im Mai 1934 noch 12 Vorzugsausgaben auf „Papier d'Arches“ kommen, von denen aber nur fünf, von 0 bis 00000 nummeriert, gedruckt wurden. Zusätzlich wurden 16 römisch nummerierte Exemplare erstellt. $\mathrm{Zu}$ den Heften gab es einen Schuber, der beidseitig mit Radierungen verziert war. Mit jedem Heft der Vorzugsausgaben wurde noch eine eigens von Max Ernst erstellte Weichgrundätzungen geliefert. ${ }^{16} 1934$ kostete die Heftreihe für Subskribenten 125 beziehungsweise 400 Francs. 

allem als Titelillustrationen gedient hatten. Sie entstammen Zeitschriften, allen voran dem Journal des beaux romans, die sich bereits völlig auf die Publikation von Romanen in Serie spezialisiert hatten. Der frühe Feuilletonroman aber war ein Serienroman, der meist ohne Illustrationen im Feuilleton abgedruckt war. Das heißt, er erschien in jenem untersten Drittel der Zeitung, das Reiseberichten, Theaterbesprechungen, Rezensionen, Glossen, Kommentaren und Unterhaltung Platz bot und durch einen deutlichen Strich vom politischen Journalismus getrennt war.

11 Die Bedeutung des unpolitischen Feuilletons wuchs mit der Einschränkung des politischen Journalismus zum Ende der Französischen Revolution. ${ }^{17}$ Waren Zeitungen bis dahin Medien kleiner Gruppen politisch interessierter Gebildeter, ${ }^{18}$ so wandten sich die Zeitungen mit dem Feuilleton an eine breitere, weniger homogene und weniger politisch interessierte Öffentlichkeit. Bedingt durch die Einführung der schneller arbeitenden, in ihren Anschaffungskosten aber teuren Rotationspressen, wurden höhere Auflagen möglich. ${ }^{19}$ Als neues Publikum boten sich die urbane Mittelschicht und das wachsende Industrieproletariat an, welches einen besseren Zugang $\mathrm{zu}$ Bildung hatten als die Landbevölkerung. Erreicht werden konnten sie jedoch erst nach einer signifikanten Preissenkung und damit einem innovativen Produktions- und Distributionskonzept, mit dem der Feuilletonroman als Mittel der „conquête systématique du marché populaire“ entstand. ${ }^{20}$

15. Juni 1836 erschien erstmalig Emil de Girardins La presse. ${ }^{21}$ Mit ihrem Preis von 40 Francs für das Jahresabonnement, statt der bisher üblichen 80, wurde die übrige Presse bald gezwungen, diesem Konzept zu folgen. Wer bestehen wollte, musste den Preis senken und wie La presse die Produktionskosten durch Annoncen decken, was wiederum eine möglichst hohe Verbreitung forderte. ${ }^{22} \mathrm{Am} 30$. September 1836 begann im Feuilleton von Le siècle die Erstveröffentlichung in Serie des Romans Patrona Calil von Alphonse Royers. Ab dem 23. Oktober wurde Balzacs La vielle fille in La presse in Serie, wenn auch nicht im Feuilleton, abgedruckt. Alfred François Nettement stellte schon 1845 fest :

Pour donner un journal à 40 ou 48 francs, il fallait donc avoir beaucoup d'annonces; pour avoir beaucoup d'annonces, il fallait avoir beaucoup d'abonnés, pour avoir beaucoup d'abonnés, il fallait trouver une amorce qui s'adressât à toutes les opinions à la fois, et qui substituât un intérêt de curiosité générale à l'intérêt politique qui groupait naguère ceux qui adhéraient au symbole d'un journal autour de leur drapeau. C'est ainsi qu'en partant de la presse à 40 francs, et en passant par l'annonce, on arrive presque fatalement au feuilleton-roman et au feuilleton immoral, deux mots pour la même idée [...].23

13 Mit der Ausweitung der Leserschichten wurde der Feuilletonroman zum Synonym für massenhaft und industriell produzierte Literatur; ${ }^{24}$ Massenware für „unbelesene“ Massen. ${ }^{25}$ Die Einteilung in Leser und des Lesens Unkundige verschob sich $\mathrm{zu}$ einer Unterscheidung zwischen Lesern mit Geschmack und einem anonymen Massenpublikum. ${ }^{26}$ Die Beziehungen zwischen „hoher Literatur" und Feuilletonroman sind dennoch zahlreich. Viele spätere Klassiker wurden erstmals im Feuilleton dem Zeitungspublikum zugänglich gemacht, anerkannte Autoren schrieben für das Feuilleton oder ließen sich von ihnen inspirieren.$^{27}$ Der Feuilletonroman selbst entnahm „hoher Literatur" Elemente, die jedoch meist auf klischeehafte Versatzstücke reduziert wurden. ${ }^{28}$ Die engste Beziehung besteht aber zwischen Serienroman, populärwissenschaftlichen Beiträgen und faits divers. 
14 Wissenschaftliche und literarische Beiträge sind in populärwissenschaftlichen Zeitschriften wie Science populaire schwer voneinander zu trennen. Neben praktischem Wissen aus Medizin oder Landwirtschaft stehen abenteuerliche Berichte von Entdeckern und Erfindern, voller spektakulärer Unfälle und Morde. Obwohl sich die populärwissenschaftlichen Zeitschriften als alternative „bonne lecture“ zur schlechten Romanlektüre präsentierten, war der Übergang $\mathrm{zu}$ den ebenfalls abgedruckten Abenteuerromanen fließend. Diese Zeitschriften waren gegründet worden, um Wissenschaft zu demokratisieren, aber Ende des 19. Jahrhunderts wurden im Angesicht zunehmender „Professionalisierung und Kommerzialisierung der Presselandschaft“ Beiträge nur noch dann abgedruckt, „,wenn sie der Logik der Medien entsprachen und die Aufmerksamkeit von Lesern und potentiellen Käufern zu wecken vermochten“..29 Die ganzseitigen Illustrationen der Titelseiten arbeiteten mit den Verfahren der Romanillustration. Sie stellten dramatische Momente dar, wie den Einsturz eines Leuchtturms oder den Tod eines Entdeckers ${ }^{30}$. Die Bildunterschriften weckten die Erwartung einer spannenden Geschichte. Es erstaunt deshalb nicht, dass Max Ernst sie gleich den Romanillustrationen für Collagen in Une semaine de bonté verwendete.

Die frühen Feuilletonromane erschienen meist ohne Illustrationen. Holzstiche fanden sich zunächst in populärwissenschaftlichen Werken, auch wurden sie für die Publikation von Sammelbänden von Feuilletonromanen angefertigt. Als erste Tageszeitung bebilderte ab 1842 die Illustrated London News ihre Nachrichten; ${ }^{31}$ vor allem mit Holzstichen, denn sie waren leicht und schnell herzustellen, abnutzungsstabil und einfach abzuändern. Bald entstand eine regelrechte „Illustrationsindustrie“; so betrieb Gustave Doré - zahlreiche seiner Illustrationen finden sich in Une semaine de bonté wieder - eine Werkstatt mit 1300 Stechern, die ausführten, was er vorgezeichnet hatte. Während es üblich war, die Druckplatten der Nachrichten von Morden, Unfällen oder Massenunruhen für Romane wiederzuverwenden, beeinflussten umgekehrt die Romanillustrationen auch die Ästhetik der Nachrichtenbilder. ${ }^{32}$

[D]ramatically lit engraving with high articulation of emotion was firmly established as a part of fiction and in social reportage. Thus the reader, in his or her experience of the discourse offered in such material, is not only presented with visual styles which may be very similar in both "fact" and "fiction" passages; he or she encounters a relationship between word and image which is fundamentally the same in both, and thus a type of discourse is shared between notional "facts" and "fiction" which colours and in many cases directs the reader's approach to the outside world. ${ }^{33}$

Die These, dass der Feuilletonroman den Blick auf die Welt prägte und das Denken seiner Epoche beeinflusste, ${ }^{34}$ wird durch seine Nähe $\mathrm{zu}$ faits divers und populärwissenschaftlichen Zeitschriften untermauert. Dieses Nebeneinander ,intensified the literary parallels between news stories, sensationally written and occasionally even fictionalized, and feuilleton novels in which crime was an ever-present element." ${ }^{35}$ So äußerte Alfred Jarry zum fait divers, „est-il autre chose, sinon qu'un roman, du moins qu'une nouvelle due à la brillante imagination des reporters?" ${ }^{36}$

Fait divers und Zeitungsroman folgten der gleichen Ästhetik der Sensationen und Spannung, umso mehr als die Fälle der faits divers oft schrittweise und in Folgen aufgeklärt wurden. ${ }^{37}$ Während der Roman narrative Muster bot, Nachrichten und Entdeckungen aufzubereiten, machte seine Nähe $\mathrm{zu}$ den faits divers und den populärwissenschaftlichen Beiträgen glaubhaft, auch im Roman ginge es um tatsächliche Begebenheiten. Die Illustrationen, als enge Verwandte der didaktischen, 
populärwissenschaftlichen Stiche, durchweg realistisch, "gestochen scharf" durch die Technik des Holzstichs, ${ }^{38}$ verliehen dem Romangeschehen zusätzliche Authentizität. Seine weite Verbreitung erlaubte es dem Roman, die Weltanschauung vieler Menschen zu prägen. ${ }^{39}$

\section{Freiheit und Ordnung des Feuilletonromans} Bedeutung des Feuilletons wuchs, welches im Gegensatz zu den bis dahin vorrangigen politischen Zeitungen weniger strenger Zensur unterlag. ${ }^{40}$ Was für die exklusiv politische Leserschaft ohne Interesse war und was sich aus moralischen und ästhetischen Gründen im Zeitungshauptteil über dem Strich verbat, fand seinen Platz im Feuilleton: Verbrechen, Skandale und der Alltag der unteren Schichten. Unbestreitbar ist zudem, dass der Feuilletonroman, der neben einem hohen Anteil an Leserinnen auch mehr als andere Bereiche der Literatur Schriftstellerinnen zugänglich war, zu einem Ort der Artikulation weiblicher Belange in der Öffentlichkeit wurde.

Wenn außerdem Literatur als „Ausdruck persönlicher und / oder gesellschaftlicher, auf jeden Fall aber historisch bedingter Hoffnungen und Illusionen, Befangenheiten und Befürchtungen " ${ }^{\text {41 }}$ begriffen werden kann, dann nicht zuletzt Romane, die in der Tagespresse veröffentlicht, darauf angewiesen waren, die täglichen Interessen eines möglichst breiten Publikums zu bedienen. ${ }^{42}$ Die Geschwindigkeit, die die wiederholten Verlängerungen einiger großer Feuilletonromanerfolge wie Sues Les mystères de Paris verlangten, „est particulièrement favorable - et c'est par là principalement qu'elle intéressa les surréalistes - à une libération des fantasmes, une explosion d'images et de scènes qui semblent parler librement le langage de l'inconscient “". ${ }^{43}$

Dennoch halte ich David H. Walkers Ansatz für vereinfachend, der in Gewalt und Exzess, die das Feuilleton prägten, den Ausdruck ungebändigter, widerständiger Realität sieht, die durch die Oberfläche der herrschenden Ordnung dringe und drohe diese zu sprengen. ${ }^{44}$ Stützt Walker sich dabei auf Foucault, ${ }^{45}$ übersieht er, dass für diesen der fait divers nicht der Disziplinierung entgegensteht, sondern ihr Mittel ist. Nach Foucault trägt der fait divers dazu bei, mitten in der Gesellschaft das Gefühl einer Gefährdung zu verbreiteten und wird so Teil einer produktiven Ordnungsmacht, die bislang unbestimmtes menschliches Leben einer Klassifikation unterwirft. ${ }^{46}$

21 Für den jungen roman feuilleton scheint die Diskussion der 1820er und 30er Jahre über einige "monströse“ Fälle bedeutend gewesen zu sein. ${ }^{47}$ Spies schreibt zu den in Une semaine de bonté verarbeiteten Illustrationen: „[D]as Hauptziel dieser Fortsetzungsgeschichten [...] liegt darin, Verbrechen zu zeigen, um zu bestrafen. Wir sind meilenweit von der kriminalistischen Metaphysik entfernt, die der ,roman noir als Bollwerk gegen die Aufklärung errichtete. ${ }^{48}$ Verbrechen wurden zum Anlass für Sanktionen, und sie wurden somit allein als Verstoß gegen die Ordnung und die Bestätigung, diese auszuweiten, wahrgenommen, nie als Ausdruck der mysteriösen Tiefe des menschlichen Wesens. ${ }^{49}$ Die bösen und monströsen Verbrecher, die in Verbindung mit kosmischen Gegenmächten stehen und die noch den roman noir bevölkern, wurden im 19. Jahrhundert zu ,individu[s] à corriger" mit abweichendem Verhalten verharmlost. ${ }^{50}$

Meist entwickelte sich die Romanhandlung um Figuren, die auf Sets markanter und bekannter Typen, „stock characters“, eingeschränkt wurden. ${ }^{51}$ Bedenkt man, dass 
zeitgleich dazu Physiognomen und Phrenologen versuchten, gefährliche Menschentypen $\mathrm{zu}$ identifizieren ${ }^{52}$ lassen sich auch in den Illustrationen nicht nur „Enzyklopädien der Gräueltaten“, ${ }^{53}$ sondern auch der Gräueltäter sehen. Diesem Gedanken folgend, kann man annehmen, dass faits divers und Feuilletonroman zu den Bestrebungen, „der Wahrnehmung der Delinquenten ein Raster aufzuzwingen“, ${ }^{54}$ beitrugen. Nach Foucault betonen die faits divers die ständige Nähe der Bedrohung und die Notwendigkeit des Kampfs gegen Kriminalität, während der Feuilletonroman das Verbrechen einem fremden Bösen zuschreibt. ${ }^{55}$ Waren die Leser also einerseits angesichts der ständigen Bedrohung durch Verbrechen bereit, die Einrichtung der Polizei im 19. Jahrhundert und die damit immer deutlichere staatliche Kontrolle des alltäglichen Lebens zu akzeptieren, so waren sie andererseits beruhigt wenn die Feuilletonromane ihnen zeigten, dass nicht sie und ihre kleinen Gesetzesübertretungen gemeint waren, sondern ganz außerordentliche Verbrechen. ${ }^{56}$

In ihrem Zusammenspiel prägen roman feuilleton und fait divers die Weltwahrnehmung; ihre spektakulären Fälle brechen die Monotonie des Alltags und unterlegen seiner Zufälligkeit eine Narration, die vor dem Chaos der Unverständlichkeit rettet, indem sie die Geschichte als mythischen Kampf von Gut und Böse erklärt; eine Narration, die die versteckten Machenschaften des Bösen aufdeckt und dem Verbrechen klar erkennbare Gesichter zuordnet. Die Illustrationen haben einen bedeutenden Anteil an der Vermittlung einer visuellen, räumlichen Ordnung.

\section{Phantasmen der Gewalt in den von Ernst verwendeten Illustrationen}

Einstürzende Brücken und Leuchttürme, Massenunruhen und Bahnüberfälle waren die Schreckens- und Faszinationsbildern eines Jahrhunderts, das von technischen und sozialen Revolutionen erschüttert wurde. Der Feuilletonroman erzählte von den unsicheren Rändern der neuen städtischen Schichten, bot seinem Publikum ein HalbweltPanorama voller Gauner und Prostituierten und gab Einblick in von moralischem Verfall bedrohte Häuser wohlhabender Aufsteiger - La cour du dragon zeigt eine solche Welt der Intrigen ${ }^{57}$ Es kann folglich behauptet werden, dass der Feuilletonroman „viel stärker von dem beherrscht ist, was er befürchtet, als von dem, was er eigentlich bewahren möchte“. ${ }^{58}$ Dabei wurde aber das überwältigend Neuartige in bekannte Schemata von Gut und Böse integriert und so die alltägliche Realität entlastet. ${ }^{59}$

Zwei Figuren der Angst und Faszination präsentiert Ernst im Collageroman : einerseits das amorphe Weibliche, ${ }^{60}$ andererseits ein männliches Individuum, das sich keinen Gesetzen beugt. Im Heft L'eau dringt Wasser in die bürgerliche Welt, zumeist geradewegs ins Schlafzimmer. Doch Blatt 23 gibt die Frage zu bedenken, ob die Zersetzung nicht genau dort ihren Ursprung nimmt, wo ein Mann den Vorhang zum Zimmer seiner Frau beiseite schiebt, aus dem die Wassermassen hervorquellen. Eine von revolutionärer Begeisterung ergriffene Masse bringt eine Brücke zum Einbruch; am Ufer hingegen ruht eine Frau - auf anderen Bildern schläft sie in wogenumtösten Betten - die zur Verkörperung der Flüsse, hysterischer Massen und elementarer Mächte des Chaos wird.

Die zweite beunruhigende und doch faszinierende Figur ist der löwenköpfige Protagonist des Hefts Le lion de Belfort. Der Löwe ist Anspielung auf Frédéric Auguste Bartholdis monumentale Löwenstatue in Belfort, welche Denkmal für den Widerstand der Stadt 
gegen die deutschen Belagerer im Krieg 1870/71 und Heroisierung der Verteidigung der französischen Zivilisationist. Der deutschen Fassung von 1963 stellt Max Ernst ein Zitat Alfred Jarrys voran, das in der französischen von 1934 fehlt : „Das hermelin ist ein unflätiges tier. An sich betrachtet ist er ein kostbares bettuch, da er aber kein zweites besitzt, so reinigt er's mit seiner zunge" ${ }^{.11}$ Die Spannung zwischen abstoßender Bestialität und erotischem Reiz ist nicht zu verkennen. Ernst polemisiert mit dem Löwen, der sich durch Orden als Vertreter von Kirche und Staat ausweist, ${ }^{62}$ und dennoch gegen ihre Moral verstößt, gegen die korrumpierte Macht der Institutionen. Und gleichzeitig zeugt Max Ernsts Blasphemie von einer viel stärkeren Auseinandersetzung mit der Faszination, die von kirchlicher und politischer Macht ausgeht, als der ,aufklärerische [...] Antiklerikalismus" der anderen Surrealisten. ${ }^{63}$

Die Faszinationskraft des Feuilletonromanhelden ist dann besonders stark, wenn er durchsakrale Elemente überhöht wird; man denke nur an Dumas' Der Graf von Monte Christo. Gleich einem Gott steht er über der Masse und den Gesetzen, die jene zähmen sollen. Das erlaubt ihm, das Böse mit dessen eigenen Waffen zu bekämpfen, ohne unmoralisch zu erscheinen. ${ }^{64}$ Gleichzeitig erlaubt dieser Superheld der zweiten Hälfte des Jahrhunderts dem Publikum um so mehr die Identifikation, als er selbst aus dem Kleinbürgertum kommend, zum Helden aufsteigt..$^{65}$ In Le lion de Belfort führt Ernst den „neuen Typ Machtmensch“ vor, ${ }^{66}$ unter dessen Löwenmaske sich der Kleinbürger versteckt. Bei ihm laufen im Schlussbild des Hefts die Fäden zusammen; drei Löwen ruhen auf Podesten mit der Aufschrift „Laudate pueri dominum“, verstreut liegen die „Embleme seiner Autorität“ ${ }^{67}$ Insignien einer militarisierten bürgerlichen Gesellschaft : Portemonnaie, Uhr, Kanonenkugeln und Orden : „die ,pueri', das sind die ehrenhaften Spießbürger, deren Kollektivportrait kalbsköpfig im Hintergrund [und Fluchtpunkt] auftaucht" ${ }^{\text {". }} 8$

Gramsci merkt an, dass die Lektüre des Feuilletonromans vor allem Kleinbürgern und kleinen Intellektuellen eine Flucht aus der Enge ihrer Lebensverhältnisse in moralisch verbrämte Gewaltphantasien erlaubte. ${ }^{69}$ Illustrationen in denen diese Phantasien und der „Helden- und Führerkult“ des späten 19. Jahrhunderts deutlich werden, sind im Heft Le lion de Belfort zusammengetragen. ${ }^{70}$ Sie lassen im Superhelden eine Präfiguration der faschistischen Version von Nietzsches Übermenschen erkennen..$^{71}$ Und so lässt sich auch das Bild Napoleons auf dem ersten Blatt des Collageromans als ein Hinweis auf den im Feuilletonroman populären Napoleonkult lesen, der nach Gramsci Vorbild für den Führerkult des italienischen Faschismus wurde. ${ }^{72}$ Max Ernst kannte die politische Situation in Italien gut, wohin er 1933/34 reiste und wo er Une semaine de bonté collagierte. Der löwenköpfige Protagonist schaut zum Bild Napoleons auf, als wolle er ihm nacheifern, während er sich von der nackten „Löwin“ abwendet, die ihn befällt; weg von der Befreiung des Tierischen hin zum paternalistischen Vorbild eröffnet er den Roman. Diese Asymmetrie verkennt Wyss, der hier einen Widerstreit zwischen „Über-Ich (General)“ und „Triebnatur (Löwe)“ sieht. ${ }^{73}$ Einige Bilder zeigen den Löwen als beschützenden Ehemann, dem Paternalismus der Superhelden entsprechend. ${ }^{74}$ Zudem schlug sich im französischen Feuilletonroman um 1900 und besonders aggressiv am Vorabend des Ersten Weltkriegs der wachsende Nationalismus nieder. Unter seinem Einfluss wurden in vielen Romanen Gewalt und Chaos bösen Verschwörern wie Juden oder Jesuiten angelastet, die die Einheit der Nation zu schwächen suchten..$^{75}$ Sie wurden von beschützenden Helden bekämpft. Blatt 32 zeigt eine Hinrichtung: Troppmann „,sous les griffes du Tigre‘ ([...] Clemenceau's nickname at the time)“: 
Clemenceau was responsible for the French negotiations at the treaty of Versailles and was thus also associated with the punitive retribution exacted upon the German people for the sake of French pride. Troppman, the Jewish criminal, signifies the outsider: widely blamed in antisemitic discourse for crime and disorder since Dreyfus. ${ }^{76}$

Auf Blatt 33, dem traurigen Schluss des Hefts, sammelt der Löwe in einer düsteren, verwüsteten Landschaft seine Opfer ein. ${ }^{77}$ Dieses Bild zeigt, was von den heroischen europäischen Nationen im Krieg übrig bleibt: „Nacht, Chaos, Flucht vor der Schuld und Totenschädel. Eine Hand weist die Richtung zur Tonne, die mit Knochen und Schädeln gefüllt, von Staatsmedaillen gestempelt, das Restprodukt der modernen Zivilisation darstellt." ${ }^{\text {"7 }}$

\title{
Die Collage von „hoher“ und „populärer“ Kunst
}

\author{
He saw his father make a painting après nature in \\ the garden [...]. Father suppressed a tree in his \\ picture, because it disturbed the "composition". \\ Then he suppressed the same tree in the garden so \\ that there was no more difference between nature \\ and art. The child felt a revolt growing in his heart \\ against candid realism and decided to direct \\ himself towards a more equitable conception of \\ the relationship between the subjective and the \\ objective world. \\ Max Ernst ${ }^{79}$
} Warenkatalogen, Klischeevorlagen und Feuilletonromanillustrationen, und spürt darin das auf, was die Ordnung unterwandert. ${ }^{85}$ Der Warenkatalog offenbart seine Verwandtschaft zur Wunderkammer. Diese widersetzt sich dem Überblick, fordert einen Blick, der sich den Dingen anpasst. Es gilt, sich dem Winzigen zu nähern, um das Riesige 
herumzugehen, unter dem sich zu ducken, was von der Decke herabhängt. Werner Spies vergleicht auch Ernsts Collagen mit den Wunderkammern: „In diesen Darstellungen der Meraviglia und der Monstren der Welt geht es nicht nur um ein Registrieren der Erscheinungswelt. Kontrastierende Kopplung, der Versuch, Ungeschautes zu präsentieren, suchen frappierende Wirkungen zu erreichen“ ${ }^{86}$ Eine „[k]ontrastierende Kopplung“ die Ernst im Warenkatalog entdeckte : „die verwirrende Gleichwertigkeit, zu der [...] der Prospekt" die Dinge zusammenzwingt, ${ }^{87}$ macht ihre Faszination aus und unterwandert dessen Ordnung. Die Zusammenstellung der Objekte der Wunderkammer erklärt sich nicht anhand von Maß und System, sondern berichtet von der Faszination, die die Objekte auf ihren Sammler ausgeübt haben.

In seinen Écritures beschreibt Ernst ein Waldgemälde seines Vaters: die Bäume „enfermée dans sa singularité (propre), et soumise pourtant à une communauté hêtreforêt $^{\text {"s8 }}$ - sind eingeschlossen in ihre Identität, von den anderen getrennt und in ihrer Anordnung entindividualisiert. ${ }^{89}$ Der Mönch, inmitten der Bäume, ist in sein Buch versenkt und gegen die Erfahrung seiner Umgebung abgeschlossen. Dies steht im Gegensatz zur kurz danach beschriebenen Erfahrung von „petit Max“, der beim Betreten des Waldes „ravissement" und „oppression“ empfindet..$^{90}$ Im Gegensatz zum Mönch kann er sich den Eindrücken des Waldes nicht verschließen.

An die Stelle eines distanzierten Blicks aufs Objekt tritt eine Berührung mit den Dingen. Die Haut, die Oberfläche, wird zur Kontaktfläche. Das Subjekt hat keine Kontrolle über die Berührungen; sie gehen von den Dingen aus. Ernst entdeckt die Arbeitsweise der Frappage, da die Bilder ihm frappierend taktil aus den Strukturen des Fußbodens entgegen treten.91 Ebenso unwillkürlich stellen sich die Sympathiebeziehungen zwischen den Katalogillustrationen ein, die zur Entdeckung der Collage führen. Auf dem Höhepunkt der Collagetechnik in den Collageromanen steigert sich dies zu „eine[r] panerotische[n] Vision. Sie kündigt von einer Sinnlichkeit, [...] die in einem Erkenntnisdrang eine Berührung mit allem Existierenden anstrebt.“92

Mittels Collage wendet sich Ernst gegen eine Kunst, der es darum geht, ein neues Universum nach den wahren Regeln der Natur zu erschaffen..$^{93}$ Statt des Schöpfens „exnihilo“, ${ }^{94}$ das das Vorgefundene leugnet, fordert er seine Transformation. In seinem Artikel über die Bricolage beschreibt Lévi-Strauss die Mechanismen mythischen Denkens anhand der Bastelei. ${ }^{95}$ Im Gegensatz zum Ingenieur, der über sein Material herrscht und es ganz auf seinen Plan hin zurichtet, begnügen sich Magier und Bastler mit zufällig Gefundenem. ${ }^{96}$ Ebenso ist Ernst Sammler, dem faszinierende „,,trouvailles ${ }^{\text {‘“ }}$ durch die dunkle Einförmigkeit der Dinge entgegenstrahlen. ${ }^{97}$

Wie sich der Bastler der „Überreste von Ereignissen“98 bedient, greift der Collageur auf den Holzstich nicht als Mittel seiner künstlerischen Produktion zurück, sondern auf Reste: gebrauchte Druckstöcke, veraltete Romane und Kataloge. Und während der Ingenieur eine wahre Natur hinter ,jene[n] Zwänge[n], die ein[en] Zivilisationszustand zum Ausdruck bringen“99 entdecken will, gibt der Bastler zu, dass Wirklichkeit immer kulturell geprägt ist, dass jedes seiner Mittel selbst eine Geschichte hat, deren Spuren sich widerspenstig im Ganzen seiner Arbeiten zeigen. Er tritt mit dem Material „in eine Art Dialog “. ${ }^{100}$

Der Dialog, zu dem die Collage herausfordert, so formulierte es Ernst in seiner dadaistischen Zeit, ist ein Streit, der Objekt und Subjekt verändert. In der DadaAusstellung von 1920 wurden zerstörte Kunstwerke mit einer Selbstverständlichkeit ersetzt, als hätten sie keinen anderen Zweck, denn, so der Begleittext zur Ausstellung, „fällt [der Besucher] dem irrwahn des antidadaismus anheim zu spät bemerkt er die personalunion von metzger und opferlamm in sich er ist dadaist schlechthin". ${ }^{101}$ Dieser 
Dialog stellt die Souveränität des betrachtenden Subjekts und die Unantastbarkeit "hoher" Kunst in Frage. ${ }^{102}$

Das zweite Bild von Une Semaine de bonté präsentiert eine Nackte, wie eine Skulptur auf einem Sockel ausgestellt. Doch der klassischen Pose widersprechen Objekte an Unterleib und Beinen, die an Prothesen erinnern. Die vielen verletzten Körper sind Hinweise auf eine Auseinandersetzung mit dem selbst erlebten Krieg am Vorabend eines neuen Kriegs. ${ }^{103}$ Dass die Prothese gegen die klassische Pose gesetzt wird, zeigt, dass die Surrealisten sich weigerten, über den Krieg hinweg zu idealisieren. ${ }^{104}$ Statt des Kriegers stellt Ernst einen verletzten Körper auf den Sockel und greift so die Überhöhung des Mannes zum Helden an, dem der Körper erste Rüstung ist. Sichtbar wird die beschämende, ,verletzte organische Substanz der Kreatur, die der Panzer verbergen sollte“ . ${ }^{105}$

Viele Figuren sind kopflos oder tierköpfig. ${ }^{106}$ Das menschliche Haupt wird entmachtet. Ihm tritt ein wuchernder Körper entgegen. Es treten Wesen mit Flügeln auf, die sich in Rasterung, Fältelung, Schwung, ja quasi als Accessoire der wallenden Kleider einfügen. Kleidung, die selbst Körperteil wird, verwächst mit der Umgebung. Wie den Mustern der Tapeten, den Stoffen, Ornamenten und Geschwülsten entsprungen, wimmeln Schlangen, Fledermäuse, Gürteltiere und Heuschrecken in Ecken und Falten. Das Monster - das zentrale Prinzip der Collage ist es, so Bauer, Mischwesen herzustellen, was mit der Aufhebung der „Identität der Personen“ einhergeht ${ }^{107}$ - steht für den zerstörten Menschen, ist aber auch Angriff auf den Idealismus, der über Zerstörung hinwegsieht, und Anschlag auf die Sexualmoral, die den Körper nach sittlichen und monströsen Berührungen einteilt, ,pour mettre en branle la machine à multiplier, pour fournir [...] aux patries des individus aptes à aux exigences de la production et au service militaire" ${ }^{108}$

Dagegen setzt Max Ernst in seiner dadaistischen Phase fantastisch collagierte Militärgeräte ohne Sinn und Zweck. In Ernsts surrealistischem Collagewerk, allem voran in den Collageromanen, finden sich keine Maschinen wieder, aber zahlreiche Kopplungen von Organischem und Anorganischem von Kreatürlichem und Technischem, die an die schizophrenen "Organmaschinen“ bei Deleuze und Guattari denken lassen. ${ }^{109}$ Die Verwandtschaft von Collageur und Bastler ist unübersehbar, die Nähe zum Wahnsinn ebenfalls. In Max Ernsts Loplop-Projekt gibt es eine Hommage an den Facteur Cheval, den Briefträger und „naiven“ Architekten Ferdinand Cheval. Das lässt sich als ein Plädoyer für die produktive Entfaltung der eigenen Obsessionen verstehen. In diesem Sinne zeigt das Schlusskapitel von Une semaine de bonté, La clé des chants, wie Frauen, die ganz offensichtlich Bildern von Wahnsinnigen entnommen sind, sich aus ihren Betten befreien, bis sie über der Erde schweben. Der Wahnsinn wird befreit und insoweit bietet das Kapitel einen Schlüssel zum Gesang und zur Kunst allgemein : Die Kunst befreit sich aus ihren allzu vernünftigen Fesseln.

Elemente der Kunst von Bastlern und „Verrückten“ werden hier der hohen Kunst angeeignet; deren Abgeschlossenheit gegenüber der niederen Kunst wird dadurch angezweifelt. Diese Grenzverletzung ist Prinzip der Collage und die Collage das bestimmende Prinzip des Collageromans. Mit der Collage wird eine Technik in die hohe Kunst eingeführt, die eine lange Tradition in der Volkskunst hat, aber ebenso lange als eine der „hohen" Kunst unangemessene Technik angesehen wurde. ${ }^{110}$ Im DADA und in der Folge im Surrealismus wird die Collage zur zentralen Technik, zur "ontologischen Bedingung fürs Werk überhaupt ".111 Mit den aufwendig gestalteten und exklusiv gedruckten Collageromanen erreicht die „Veredelung“ der Collagetechnik ihren Höhepunkt, wobei gleichzeitig alles Edle der „hohen“ Kunst in Frage gestellt wird. 


\section{Fazit : Unordnung}

Wenn uns Max Ernst die Bilder vor Augen führt, von denen das Denken der Generation seines Vaters geprägt war, dann heißt es nicht, dass er die Ordnung der Zeit um 1900 restaurieren will. Die Gewalt der Feuilletonromanillustrationen war sehr wohl Teil dieser Ordnung, und so ist es verständlich, dass die Surrealisten auch das Morden des Krieges „la plus grandiose manifestation de la folie moderne“" ${ }^{{ }_{112}}$ - nicht in einem Ausbruch des Irrationalen, sondern in dieser Ordnung, die von gewaltsamen Helden und ihren Anhängern aufrecht erhalten werden sollte, begründet sahen.113

Der surrealistische „Kult des Bösen" ${ }^{\text {"114 }}$ sollte die Einteilung in gute und böse Gewalt unterlaufen. Max Ernst wendet sich gegen einen Krieg, der im Namen von Fortschritt und Freiheit geführt wird : „Indem er die Gräuel vor Augen führt, die im Dienst an diesen Idealen begangen werden, fordert Max Ernst das Eingeständnis, daß die wirklichen Gewalttäter die sind, die die Macht ausüben und verlangt nach der Gegengewalt“ ${ }^{115}$ Den guten Gewalten setzen die Surrealisten die monströse, Unordnung stiftende Collage entgegen. Sie respektiert die Grenzen zwischen Mensch, Tier und Maschine, „hoher“ und „niedriger" Kultur, Gut und Böse nicht.

So sehr Une semaine de bonté zu solchen Überlegungen anregen kann, so wenig wird der Collageroman dadurch entschlüsselt. Wer ihn zu lesen versucht, wird irritiert werden, durch Assoziationen abgelenkt zurückblättern und das Buch entnervt weglegen. Der Collageroman stößt sein Publikum immer wieder darauf, dass er sich nicht auf eine vorgefasste Idee eines organisierenden Subjekts hin auflösen lässt; umso mehr bleiben Intertextualität und Materialität präsent.

Der Collageroman bildet eine materiell fassliche Entität in einem Netz von Beziehungen, eine „petite machine“, die im Zusammenhang mit anderen steht; zu Organmaschinen, denn die Hand, die über das Papier streicht, interagiert sehr wohl auch sinnlich: „how autoerotic is such rubbing?“",116 Ernst hätte wohl nichts dagegen. Ist der Roman also eine „machine d'amour“, oder eine „machine révolutionnaire“?117 Vielleicht. Collage, so Aragon 1921, ist „une machine à bouleverser le monde ${ }^{{ }^{118}}$. Rosalind Krauss entwirft das Bild Adornos, der während seines Rückblicks auf den Surrealismus einen Collageroman auf seinem Schoß liegen hat. ${ }^{119}$ Die Schwere des Buches, das Berühren des Papiers, ist nicht ohne Bedeutung.

Im Gegensatz zu Plakat, Zeitschrifttitelbildern oder Film ist ein solch technisch aufwendig gestaltetes Buch jedoch wenig geeignet, die große Menge zu erreichen und politisch zu agitieren. Stattdessen lädt der Collageroman den Bibliophilen ein, die Welt um sich herum zu vergessen. In der Wahl der Romanform liegt eine Abkehr von der „WeisungsSprache “"120 linker und rechter Avantgarden. Angeregt wird, die Mythen und Phantasmen zu hinterfragen, die der eigenen politischen Überzeugung zu Grunde liegen mögen. Une semaine de bonté schlägt vor, sich auf die Denkweise von Mythen und Phantasmen einzulassen, unterläuft sie zugleich und entsetzt damit die gegenwärtige Ordnung, denn wer die Bilder betrachtet, findet nicht sofort die passende Schublade der Verdrängung.

Wer die Lektüre des Collageroman auf sich nimmt, schafft keine neue Welt, erreicht aber auch keine Phantasiewelt in die zu flüchten möglich wäre. Wer den Collageroman auf einen Sinn hin auflösen will, wird darauf stoßen, dass hier ein Netz von Bezügen gespannt wird, die sich gegenseitig verfremden und letztlich nur durch das Buch 
zusammengehalten werden. Als materielle Spuren sind sie präsent, in ihrer Sinnfülle sind sie noch lange nicht vollständig erschöpft. Auch eine eindeutige politische Aussage hält der Collageroman nicht bereit. Kunst, so Ernst, kann den politischen Kampf nicht ersetzen. ${ }^{121}$ In seiner Sperrigkeit ist das Material Ausdruck einer Welt, die dem, der auf Verwertung drängt, fremd geworden ist. Es wird klar, Une Semaine de bonté ist nichts anderes als eine dadaistische Maschine in Buchform : Sie würde niemals funktionieren.

\section{NOTES}

1. ERNST, Max 1934. Une semaine de bonté ou Les sept éléments capitaux. Paris. Das Werk erschien in den Éditions Jeanne Bucher und wurde bei Georges Duval gedruckt. Die deutsche Erstausgabe erschien im Gerhardt Verlag in Berlin: ERNST, Max 1963. Une semaine de bonté oder Die weiße Woche. Ein Bilderbuch von Güte, Liebe und Menschlichkeit. Berlin.

2. ADAMOWICZ, Elza 1998. Surrealist Collage in Text and Image. Dissecting the Exquisite Corps. Cambridge, 13.

3. Der Titel Une semaine de bonté spielt einerseits auf die christliche Schöpfungsgeschichte an, andererseits auf eine zeitgenössische Wohltätigkeitsvereinigung mit dem Namen „La semaine de la bonté“, gegründet 1927, so die Vorankündigung vom 6. 6. 1927 in Le Figaro, „de créer en dehors de toute opinion politique ou religieuse, un vaste mouvement d'idée en faveur de tout être dépendant ou faible“. Wenn man in Ume semaine de bonté eine Kritik am Zustand der französischen Gesellschaft sieht, dann auch an einer solchen „unpolitischen“ und unkritischen Haltung gegenüber gesellschaftlichen Missständen, die ganz auf die Kraft der menschlichen Güte vertraut und auf Gewalt und Diskussion verzichten will, wie es in einem Artikel in Le Figaro vom 23. 5. 1928 heißt. Der Untertitel der deutschen Ausgabe macht diese Kritik explizit. Die Ausgaben von Le Figaro als Digitalisat unter http://gallica.bnf.fr/ark:/12148/bpt6k2951580.langFR und http://gallica.bnf.fr/ark:/12148/bpt6k295513z.langFR.

4. Die Zitate der französischen Erstausgabe stammen von Marcel Schwob, Hans Arp, den Ärzten Jean-Ovide Decroly und Raymond Buyse, Paul Éluard, André Breton und dem Feuilletonromanautor Joseph-Pétrus Borel d'Hauterive. Die Überschriften der deutschen Erstausgabe wurden von Max Ernst übersetzt, Überschriften und einleitende Zitate von ihm überarbeitet. Hinzu kam zum Beispiel das dem ersten Heft voransgestellte Zitat Alfred Jarrys.

5. Spies wendet sich gegen eine Interpretation, die die Inhalte der verwendeten Feuilletonromane einbezieht. SPIES, Werner 1974. Max Ernst. Collagen, Inventar und Widerspruch. Köln, 9f.

6. ADORNO, Theodor W. 1981. „Rückblickend auf den Surrealismus“. In: Ders. Noten zur Literatur. Frankfurt a.M., 100-105, 103.

7. BRETON, André 1962. „Anweisung für den Leser“. In: Max Ernst. La femme 100 têtes. Berlin, (ohne Seitenzahlen, Seiten 11-15, Seite 11). Siehe auch GILLET, Michel 1983 „Machines de romans-feuilletons“. In: Romantisme. Revue du dix-neuvième siècle. Bd. 13. Nr. 41, 79-90, 79. Als Digitalisat auch unter http://www.persee.fr/web/revues/home/prescript/article/ roman_0048-8593_1983_num_13_41_4656.

8. THIESSE, Anne-Marie 1984. Le Roman du quotidien. Lecteurs et lectures populaires à la Belle Époque. Paris, 83. Siehe zudem QUEFFÉLEC, Lise 1989. Le Roman-feuilleton français au XIXe siècle. Paris, 3. 
9. LETHEN, Helmut 1994. Verhaltenslehren der Kälte. Lebensversuche zwischen den Kriegen. Frankfurt a.M., 36 u. 196.

10. BAUER, Gerd 1977. „Max Ernsts Collageroman ,Une Semaine de Bonté““. In: Wallraff-RichartzJahrbuch. Westdeutsches Jahrbuch für Kunstgeschichte. Bd. XXXIX. 237-257, 251.

11. REY-DEBOVE Josette, Alain Rey (Hg.) 2000. Le nouveau Petit Robert. Paris, 337.

12. QUEFFÉLEC 1989, 89.

13. BONNET, Éric 2008. „Rêves et figures emblématiques, Une semaine de bonté ou Les sept éléments capitaux“. In: Julia Drost, Ursula Moureau-Martini, Nicolas Devigne (Hg.) 2008. Max Ernst, l'imagier des poètes. Paris, 117-128, 120.

14. SKIRA, Albert, TERIADE 1934. Minotaure. Nr. 5. Paris, (ohne Seitenzahlen, Seite 22 des Anhangs).

15. Siehe zu den Umständen der Veröffentlichung SPIES, Werner 2008.1. „Die Desaster des Jahrhunderts“. In: Max Ernst Une semaine de bonté. Die Originalcollagen. Hg.: Werner Spies. Köln, 10-71.

16. Die Weichgrundätzungen wurden im Atelier 17 von Stanley William Hayler in Paris gedruckt.

17. ATKINSON, Nora 1929. Eugène Sue et le Roman-feuilleton. Thèse pour le Doctorat d'Université. Nemours, 5.

18. HABERMAS, Jürgen 1979. Strukturwandel der Öffentlichkeit. Untersuchungen zu einer Kategorie der bürgerlichen Gesellschaft. Darmstadt, Neuwied, 74.

19. AUBRY, Danielle 2006. Du Roman-feuilleton à la série télévisuelle. Pour une rhétorique du genre de la sérialité. Bern u.a., 28.

20. EBD., 17.

21. Digitalisat unter http://gallica.bnf.fr/ark:/12148/bpt6k426719v.langFR

22. ATKINSON 1929, 7.

23. NETTEMENT, Alfred François 1845. Études critiques sur le roman feuilleton. Paris, 2f.. Digitalisat unter https://archive.org/details/tudescritiques01nettuoft.

24. Um dem enormen Bedarf an Fortsetzungen nachzukommen, betrieb manch einer - Alexandre Dumas père war dafür berüchtigt - arbeitsteilige Schreibwerkstätten. Saint-Beuve sprach 1839 als erster von industrieller Literatur. Siehe AUBRY 2006, 1. Thackeray warf Sue „works of calculation and trade“ vor. GRUBITZSCH, Helga (Hg.) 1977. Materialien zur Kritik des FeuilletonRomans.,Die Geheimnisse von Paris“ von Eugène Sue. Wiesbaden, 15.

25. QUEFFÉLEC 1989, 5.

26. CHARTIER, Roger, Henri-Jean MARTIN (Hg.) 1985. Histoire de l'édition française, Bd. 3. Le temps des éditeurs. Du Romantisme à la Belle Époque, Paris, 469.

27. So beeinflusst Les mystères de Paris (1842) von Sue eine ganze Reihe von Mystères bis hin zu Les mystères de Marseille von Zola (1867), aber auch Hugos Misérables (1862). Siehe dazu GRUBITZSCH 1977, 1f.

28. ECO, Umberto 1989. „Eugène Sue: ,Sozialismus und Trost.“ In: Ders. Apokalyptiker und Integrierte. Zur kritischen Kritik der Massenkultur. Übers. Max Looser. Frankfurt a.M., 12.

29. GALL, Alexander 2011. „Authentizität, Dramatik und der Erfolg der populären zoologischen Illustration im 19. Jahrhundert. Brehms Thierleben und die Gartenlaube“. In: Stefanie Samida (Hg.). Inszenierte Wissenschaft.Zur Popularisierung von Wissen im 19. Jahrhundert. Bielefeld, 103-126, 121.

30. Das im Collageroman verwendete Bild mit dem einstürzenden Leuchtturm ist das Titelbild von La science populaire vom 22. April 1880. Digitalisat unter http://gallica.bnf.fr/ark:/12148/ bpt6k54245185.image.langFR.r

31. WARLICK, Marjorie Elizabeth 1984. Max Ernst's Collage Novel Une semaine de bonté.Feuilleton Sources and Alchemical Interpretation. Maryland, 72.

32. EBD. 56.

33. SILLARS, Stuart 1995. Visualisation in Popular Fiction 1860-1960. Graphic Narratives, Fictional Images. London, New York, 33. 
34. HÜLK, Walburga 1985. Als die Helden Opfer wurden. Grundlagen und Funktion gesellschaftlicher Ordnungsmodelle in den Feuilletonromanen "Les Mystères de Paris“ und „Le Juif errant" von Eugène Sue. Heidelberg, 58. Siehe auch QUEFFÉLEC 1989, 13.

35. WARLICK 1984, 56. Siehe auch THIESSE 1984, 107.

36. Alfred Jarry nach WALKER, David H. 1995. Outrage and Insight. Modern French Writers and the 'Fait Divers'. Oxford, Washington D.C., 19.

37. Siehe QUEFFÉLEC 1989, 29. WARLICK 1984, 56.

38. Diese Genauigkeit des Holzstichs ist der Grund, weshalb viele Zeitungen noch Ende des 19. Jahrhunderts den Stich der fotografischen Abbildung vorziehen.

39. Siehe THIESSE 1984, 24: „[C]e qui caractérise avant tout le roman-feuilleton, [...] c'est cette apparence de bien gratuit": Die Romanserie fand umso leichter Eingang auch in sparsame Haushalte, als die Zeitschrift vorrangig, was als Vorwand dienen konnte, dem Mann politische Information, der Frau praktische Ratschläge und moralische Erbauung bot. Anders als Bücher, die dauerhafte Konzentration erfordern, fügte sich der leicht zu lesende und täglich beschränkte Zeitungsroman in die kurzen Pausen des Arbeitstags der Hausfrau. Da sich lange die Tradition der „lecture à haute voix“ erhielt, erreichte der Feuilletonroman auch viele Menschen, die des Lesens unkundig waren. Siehe auch ECO, Umberto 1990. Il superuomo di massa. Retorica e ideologia nel romanzo popolare. Mailand. Und AUBRY 2006, 14.

40. ATKINSON 1929, 5.

41. NEUSCHÄFER, Hans-Jörg. 1976. Populärromane im 19. Jahrhundert. Von Dumas bis Zola. München, 22.

42. NEUSCHÄFER, Hans-Jörg, Dorothee FRITZ-EL AHMAD, Klaus-Peter WALTER 1986. Der französische Feuilletonroman. Die Entstehung der Serienliteratur im Medium der Tageszeitung. Darmstadt, 12.

43. QUEFFÉLEC 1989, 30. Siehe auch GILLET 1983, 79.

44. WALKER 1995, 1f.: „As society increasingly represses individual idiosyncrasies in favour of consensual values, frustrated impulses seek displaced gratification. [...] [I]ncidents which occur in the bypass of every day life and do not find their way into the mainstream of public affairs may owe their marginal status not to any intrinsic triviality but to the fact that the dominant ideology cannot accomodate them properly."

45. EBD., 4.

46. FOUCAULT, Michel 1977. Überwachen und Strafen. Die Geburt des Gefängnisses. Übers. Walter Seitter. Frankfurt a.M., 369.

47. FOUCAULT, Michel 1999. Les Anormaux. Cours au Collège de France. 1974-75. Hg. Valerio Marchetti, Antonella Salomoni. Paris 57f.

48. SPIES, Werner 2008.2. „Dream and Revolution. On the Exhibition“. In: Ders. Dream and Revolution. Ostfildern, 30.

49. Siehe auch ECO 1990, 15.

50. EBD., 53f., $68 \mathrm{f}$.

51. Siehe WARLICK 1984, 49. THIESSE 1984, 140. ECO 1990, 11.

52. GILLET, Michel 1983. „Machines du roman feuilleton.“ In: Romantisme. Nr. 41. 79-90, 81. Digitalisat unter: http://www.persee.fr/web/revues/home/prescript/article/ roman_0048-8593_1983_num_13_14_4656. Siehe auch GRUBITZSCH 1977, 9: „Sue [stattet] die Verbrecher nach Art der Phrenologen mit physischen Merkmalen aus, die ihre naturbedingte Anlage zum Verbrechen zum Ausdruck bringen“.

53. SPIES 1974, 179.

54. FOUCAULT, Michel 1977. Überwachen und Strafen. Die Geburt des Gefängnisses. Übers.: Walter Seitter. Frankfurt a.M., 369.

55. EBD.

56. Siehe AUBRY 2006, 67. QUEFFÉLEC 1989, 65. 
57. NEUSCHÄFER 1976, 33. AUBRY 2006, 15.

58. NEUSCHÄFER, FRITZ-EL AHMAD, WALTER 1986, 14.

59. NEUSCHÄFER 1976, 33.

60. Ernst arbeitet heraus, wie sehr die Feuilletonromanillustrationen dem voyeuristischen Blick der (männlichen) Leser Attraktionen bieten, indem sie neben Gewalt- und Unfallszenen vor allem weibliche Körper in lasziven Posen ausstellen. Ernst entblößt die Frauen konsequent und macht so ihre Funktion als Lustobjekte eindeutig. Oft karikiert er die scheinbar unbeteiligte Distanz des Voyeurs, indem er in die Ferne blickende Entdecker gleich Zoobesuchern auf schlafende Frauen niedersehen lässt. Oder er zerstört die Distanz, indem er den Voyeur mit dem Objekt verstrickt. Siehe auch LINDNER, Ines 2008. „Economie technique et effets surréels. Stratégies de montage dans Une semaine de bonté “. In: Julia Drost, Ursula Moureau-Martini, Nicolas Devigne (Hg.) 2008. Max Ernst, l'imagier des poètes. Paris, 121-144, 131. Ines Lindner legt dies anhand eines Vergleichs des vierten Blatts aus „L'île de pâques“ mit seiner Vorlage aus Jules Marys Les Damnées de Paris dar. Eine junge Frau umarmt einen Soldaten. Sie blickt ihm nicht ins Gesicht, sondern über seine Schulter, in Richtung des Betrachters gedreht, ins Leere. So entblößt sie ihre Brust den Blicken des Voyeurs. Ernst stellt nun an die Seite des Soldaten eine Figur mit Fetischkopf. Sie fasst an den Ausschnitt der Frau „[et] fait un usage palpable de ce qui est présenté à l'observateur“. EBD.

61. ERNST 1963, (ohne Seitenzahlen, Seite 5).

62. BAUER 1977, 242.

63. SPIES 1974, 181.

64. NEUSCHÄFER 1976, 15. Man kann sich in Dumas' Le Comte de Monte Cristo einerseits über die Störung des „Harmoniebedürfnis des bürgerlichen Edmond Dantès“ empören, sich mit ihm als Opfer identifizieren, und sich andererseits an der unmäßigen Gewalt ergötzen, mit der er, zum Grafen aufgestiegen, sich an seinen Peinigern rächt. Siehe auch NEUSCHÄFER, FRITZ-EL AHMAD, WALTER 1986, 218. Sie schreiben über den Graf von Monte Cristo: „Seine Gottähnlichkeit ist durch quasi christologische Wandlung gerechtfertigt und das Schicksal gibt ihm Recht, wo er ohne Rücksicht auf menschliches Gesetz im Kampf für das Gute vorgeht." Siehe dazu auch Max Stirner, der in seiner 1842 erschienenen Rezension Eugène Sues Mystères klagt: „Ihr, die ihr die Sünder bekehren und bessern wollt, ihr seid ja selbst unbekehrbar und unverbesserlich. Ihr lasst den Zweifel gar nicht an euch kommen, ob das Gute nicht eben ein - leerer Wahn sei“. STIRNER, Max 1976. „Die Mysterien von Paris, von Eugene Sue“. In: Ders. Kleinere Schriften und seine Entgegnungen auf die Kritik seines Werkes „Der Einzige und sein Eigentum“. Stuttgart, 278-295 1976, 279. Max Ernst studierte Stirner ausführlich. Siehe WARLICK, Majorie Elizabeth 2001. Max Ernst and Alchemy, A Magician in Search of Myth. Austin, 34.

65. Siehe NEUSCHÄFER 1976, $35 f$.

66. WYSS, Dieter 1950. Der Surrealismus. Eine Einführung und Deutung surrealistischer Literatur und Malerei. Heidelberg, 66.

67. EBD., 72.

68. EBD., $72 f$.

69. Siehe HAUG, Wolfgang Fritz 2006. Einführung in Marxistisches Philosophieren. Hamburg, 162. NEUSCHÄFER 1976, 42.

70. EBD., 37.

71. So lässt sich auch Gramsci verstehen, der weniger den Nietzsches Philosophie vom Übermenschen verurteilen will, als zu Vorsicht gegenüber dem vermeintlichen Anhänger mahnt, bei dem es „angebracht [ist], zu fragen [...], ob seine Konzeption des „Übermenschlichen“ [...] rein nietzscheanischen Ursprungs sind [...] oder ob sie viel bescheidenere Ursprünge haben, beispielsweise eine Verbindung mit der Fortsetzungsliteratur“. GRAMSCI, Antonio 1991. Marxismus und Literatur. Hg. u. übers. Sabine Kebir. Hamburg, 222. Eco erkennt hier einen Verweis Gramscis auf Mussolini, der selbst Feuilletonromanautor war. ECO 1990, VI. 
72. GRAMSCI, Antonio 1992. Gefängnishefte. Bd. VIII. Hg. u. übers. Klaus Bochmann, Wolfgang Fritz Haug u.a. Hamburg, 1829. NEUSCHÄFER 1976, 37. Dorothee Fritz-El Ahmad in NEUSCHÄFER, FRITZ-EL AHMAD, WALTER 1986, 128f. Ernsts Collageroman ist sicher eine Reaktion auf die nationalsozialistische Machtübernahme, setzt sich aber auch mit Eindrücken der Reise auseinander, die Ernst durch das seit 1922 faschistische Italien führte. Der Collageroman entstand im Rahmen dieser Reise auf dem Schloss der Duchesse de Gramont in Vigoleno.

73. WYSS 1950, 65.

74. ECO 1990, 13. NEUSCHÄFER, FRITZ-EL AHMAD, WALTER 1986, 218.

75. ECO 1990, 75. NEUSCHÄFER, FRITZ-EL AHMAD, WALTER 1986, 91f. und 142.

76. BAKER, Simon 2007. Surrealism, History and Revolution. Bern, $58 \mathrm{f}$.

77. BAUER 1977, 242.

78. WYSS 1950, 72.

79. ERNST, Max 1948. Beyond Painting. New York, 28.

80. BAUER 1977, 242.

81. Max Ernst nach SPIES 1974, 95.

82. EBD.

83. Siehe ERNST, Max 1970. Écritures. Paris, 16.

84. BRETON, André 1968. Die Manifeste des Surrealismus. Übers. Ruth Henry. Reinbek bei Hamburg, $14 \mathrm{f}$.

85. Siehe ERNST, Max 1970, 31: „[L]e catalogue d'un fournisseur d'articles scolaires attire son attention. [...]. Éléments de nature si diverse que l'absurdité qui se dégageait de leur accumulation trouble son regard et ses sens; suscite des hallucinations et donne aux objets représentés des sens nouveaux que changent rapidement.“

86. SPIES, Werner 1982. Max Ernst - Loplop, Die Selbstdarstellung des Künstlers. München, 38. Siehe auch ADAMOWICZ 1998, 43.

87. SPIES 1974, 55.

88. ERNST 1970, 15.

89. Max Ernst war im Ersten Weltkrieg Artillerist. Der gerasterte Wald lässt somit auch an den Wald im Fadenkreuz denken. Seine eigenen Waldbilder, in denen die Bäume nur noch Splitter sind, zeigen die Zerstörung durch den Krieg. Siehe LADLEIF, Christiane 2003. Die Zerstörung des Auges. Ein Motiv des Surrealismus im Kontext der Histoire de l'œil George Batailles. Weimar, 24.

90. ERNST 1970, 15.

91. EBD., 242.

92. SPIES 1982, 52f. Deshalb lud Ernst Spies immer wieder „dazu ein, mit den Fingerspitzen die Collagen abzutasten, sie wie Blindenschrift zu entziffern." SPIES 2008.1, 22.

93. ERNST, Max 1970.1. Die Nacktheit der Frau ist weiser als die Lehre des Philosophen. La nudité de la femme est plus sage que l'enseignement du philosophe. Köln, Paris, (ohne Seitenzahlen, Seite 37).

94. EBD., (Seite 5f.).

95. Mit seinem Bricolage-Aufsatz erhellt Lévi-Strauss das Prinzip der Collage angemessener als in seinem Essay zu Max Ernst und dem Surrealismus. Zur Bricolage siehe LÉVI-STRAUSS, Claude 1994. Das wilde Denken. Übers.: Hans Naumann. Frankfurt a.M. Zum Surrealismus DERS. 2008. „Meditative Malerei“. In: Ders. Der Blick aus der Ferne. Frankfurt a.M., 355-360.

96. LÉVI-STRAUSS 1994, 30.

97. EBD., 401. Ernst vergleicht sich auch mit einem blinden Schwimmer. Siehe ERNST 1970, $244 f$.

98. LÉVI-STRAUS 1994, 30.

99. EBD. 33.

100. EBD. 31. Siehe auch EBD. 33ff.: Das Ergebnis ist deshalb „immer ein Kompromiß [... Wenn das Projekt des Bastlers] einmal verwirklicht ist, wird es also unvermeidlich gegenüber der ursprünglichen Absicht verschoben sein, ein Effekt, den die Surrealisten zutreffend ,objektiven Zufall genannt haben." Der Collageur kann den bestehenden Sympathiebeziehungen des 
Materials nie ganz ausweichen. Wie Ähnlichkeiten die Zusammenstellungen bestimmen, lässt sich auf Blatt $29 \mathrm{im}$ Heft La cour du dragon nachvollziehen, wo sich an eine Frau auf einem Sofa mit kassettenförmig abgestepptem Überzug ein dem Muster ähnlich geschupptes Gürteltier schmiegt.

101. ERNST 1970, 35.

102. ADORNO 1981, 102.

103. SPIES, Werner 2005. „Nightmare and Deliverance“. In: Ders., Sabine Rewald (Hg.). Max Ernst. A Retrospective. New York, 21-35, 30.

104. Siehe auch STICH, Sidra (Hg.) 1990. Anxious Visions, Surrealist Art. New York, 33.

105. LETHEN 1994, 246.

106. An vielen Stellen von Une Semaine de bonté taucht die kopflose Frau auf, der Max Ernst mit La femme 100 tête, gelesen cent oder sans, ein eigener Collageroman gewidmet hat.

107. BAUER 1977, 253f.

108. ERNST 1970, 184.

109. DELEUZE, Gilles, Felix GUATTARI 1977. Anti-Ödipus. Kapitalismus und Schizophrenie 1. Übers. Bernd Schwibs. Frankfurt a.M., 8.

110. Noch Helga Wescher schreibt: „Als Produkte von Kunsthandwerk, Volks- und Liebhaberkunst bleiben alle diese Vorarbeiten [die Collagen der volkstümlichen Tradition] auf den Seitenlinien der künstlerischen Entwicklung und üben auf sie [die Collagen der anerkannten Künstler] keinen Einfluß aus.“ Wescher nach SPIES 1974, 12.

111. EBD., 11. Die surrealistischen Collagen der Collageromane unterscheiden sich von den Collagen der dadaistischen Zeit insoweit, als anstatt der „synthetischen Collagen“, bei denen einzelne Elemente zu einem neuen Bild gefügt werden, die „analytische Collage“ überwiegt. Sie „geht von einem übergeordneten rahmenbildenden Kontext aus. Er wird sozusagen zum Aufenthaltsort, an dem sich die Begegnung und Durchdringung disparater Elemente abspielt" EBD., 105. Hierfür waren die ganzseitigen Illustrationen der Feuilletonromane besonders geeignet.

112. ERNST, 1970, $24 \mathrm{f}$.

113. ELUARD, Paul 1948. „Beyond Painting“. In: Max Ernst. Beyond painting. New York, 191, 191.

114. BENJAMIN, Walter 1977. Gesammelte Schriften. Bd. II.1. Hg. Rolf Tiedemann, Hermann Schweppenhäuser. Frankfurt a.M., 1977. Band II.1, 304.

115. BAUER 1977, 243.

116. STORR 2005, 53.

117. DELEUZE, GUATTARI 1980, 10.

118. Louis Aragon (1921) nach ADAMOWICZ, 1998, 13.

119. KRAUSS, Rosalind 1993. The optical unconscious. Cambridge/Mass., London, 34.

120. LETHEN 1994, 213.

121. SPIES, Werner 2000. Max Ernst - Die Rückkehr der Schönen Gärtnerin 1950-1970. Köln, 20. 